\title{
Fabrication and Electrical Characterization of Multiwalled Carbon Nanotube-Based Circuit at Room Temperature
}

\author{
Yitian Peng, ${ }^{1,2}$ Yuanzhong $\mathrm{Hu}^{3}$ and Weibing $\mathrm{Lu}^{4}$ \\ ${ }^{1}$ Jiangsu Key Laboratory of Design and Manufacture of Micro/Nano Biomedical Instrument, Southeast University, \\ Nanjing 211189, China \\ ${ }^{2}$ State Key Laboratory of Mechanical Transmission, Chongqing University, Chongqing 400044, China \\ ${ }^{3}$ State Key Laboratory of Tribology, Tsinghua University, Beijing 100084, China \\ ${ }^{4}$ State Key Laboratory of Millimeter Waves, School of Information Science and Engineering, Southeast University, \\ Nanjing 211189, China
}

Correspondence should be addressed to Yitian Peng, pengyitian@gmail.com

Received 27 March 2010; Revised 25 April 2010; Accepted 17 May 2010

Academic Editor: Jianyu Huang

Copyright ( $\odot 2011$ Yitian Peng et al. This is an open access article distributed under the Creative Commons Attribution License, which permits unrestricted use, distribution, and reproduction in any medium, provided the original work is properly cited.

\begin{abstract}
Multiwalled carbon nanotube (MWCNT) deposited on a pair of predetermined aluminum electrodes treated with the (3-Aminopropyl)-triethoxysilane (APTES) self-assembled monolayers (SAMs). The MWCNT bridges electrodes and forms electrode/MWCNT/electrode circuit on silicon with $500 \mathrm{~nm}$ silicon dioxide. Then the Metal (Ti/Au) pads were fabricated on MWCNT to bury the MWCNT into metal electrodes. The electrical properties of MWCNT-based circuits before and after the fabrication of metal pads were characterized. Results indicate that metal pads on MWCNT improved the electrical properties MWCNT-based circuit largely.
\end{abstract}

\section{Introduction}

Carbon nanotubes (CNTs) have exhibited a rich variety of intriguing electronic properties, such as metallic and semiconducting behavior, exhibiting ballistic transport along the tubes, and sustaining current density as high as $10^{9} \mathrm{~A} / \mathrm{cm}^{2}$. These properties suggest that CNTs are potential candidates for nanoelectronic applications such as field-effect transistors, electrical interconnect, and sensors. Nevertheless, difficulties in handling an individual CNT and positioning it at a desired location hamper the absolute exploration of CNT properties and also devices for practical applications. Large efforts are underway worldwide to address these challenges. Early CNT devices were fabricated by depositing CNTs from liquid suspension randomly, either on top of prepatterned electrodes or directly onto a substrate prior to electrode patterning [1]. This process has the advantages of low cost, scalability and compatibility with many kinds of substrate materials. However, it has significant disadvantage with too much randomness on the placement of CNT. High-density selective placement methods for CNTs have been developed by preparing the aminopropyltriethoxysilane(APTES) SAMs on the prepatterned electrodes $[2,3]$. However, the contact resistance between the CNTs and electrodes is too big and hinder the effective connection between the CNT and the external world. It means that the MWCNT is not easy to function as an active component in device $[4,5]$. In this paper, multi-walled carbon nanotubes (MWCNTs) were placed on prepatterned aluminum electrodes by chemical method using self-assembled monolayers and the metal pads were fabricated on MWCNT to improve the electrical contact between electrodes and MWCNT. Electrical properties of MWCNT-based circuits with and without metal pads on MWCNT were investigated.

\section{Experiment and Analysis}

The MWCNTs used in our study were obtained from Shenzhen Nanotech Port Co. Ltd in China. The purity of MWCNTs is greater than $95 \%$. The axial dimension and diameter of the MWCNTs were 5-15 $\mu \mathrm{m}$ and $10-20 \mathrm{~nm}$, respectively. The MWCNT suspensions were prepared by 


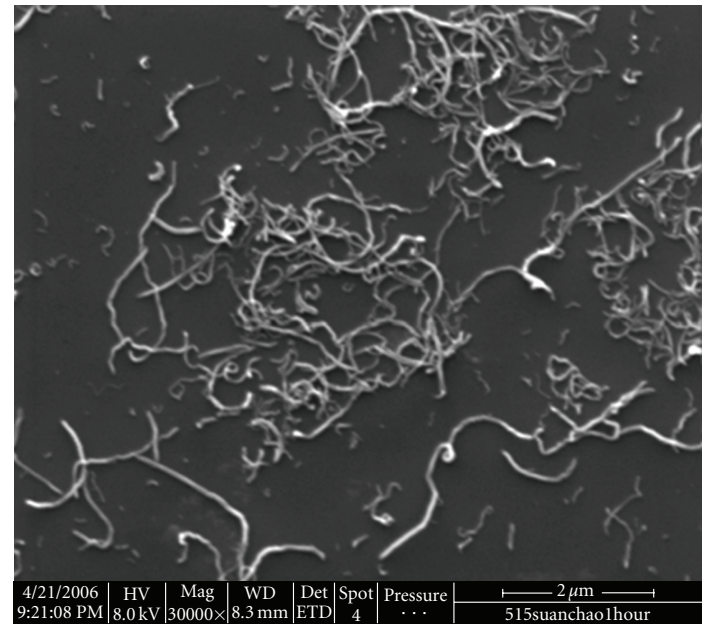

(a)

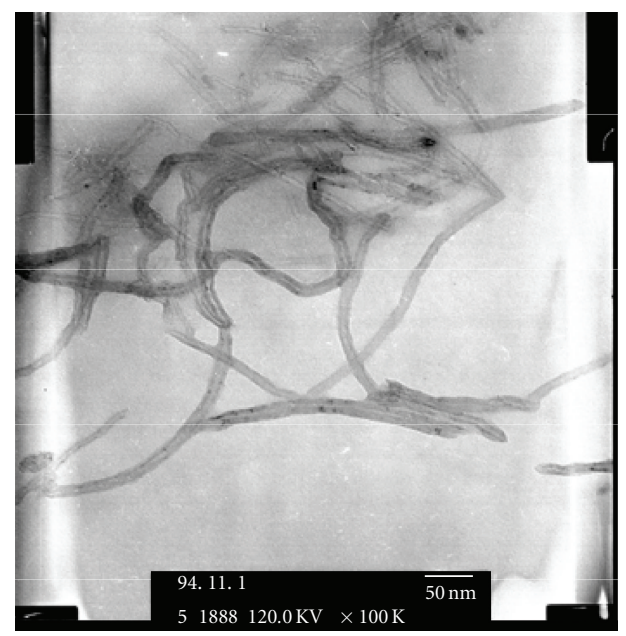

(b)

FIgURE 1: SEM (a) and TEM (b) image of dispersed MWCNTs.

ultrasonicating the MWCNT in dimethylfloramine (DMF) solution for half an hour. The MWCNT suspension was centrifuged to remove large particles and CNT bundles. To examine the dispersion of MWCNTs in DMF solution, we dropped the dispersion onto a copper grid and silicon substrate, while being dried by nitrogen gas. The configuration of dispersed MWCNTs was observed through Transmission electron microscope (TEM, JEM-200CX) and Field-emission scanning electron microscope (FESEM). The representative TEM and SEM image is showed in Figure 1, from which we can see the MWCNTs were well dispersed appropriate to the fabrication of MWCNT-based circuits.

A scheme for the deposition of MWCNTs on patterned aluminum electrodes with self-assembled monolayers is illustrated in Figure 2(a). Lightly n-doped Silicon (100) wafer with $500 \mathrm{~nm}$ of thermal dioxide silicon was used as the substrate for the fabrication of patterned electrodes. The substrate was cleaned as follows: firstly they were ultrasonicated in acetone and ethanol for 10 minutes each and then immersed into a Piranha solution at $80^{\circ} \mathrm{C}$ for 30 minutes.
To fabricate the patterned metal electrodes, the silicon wafers were metalized by evaporating $60 \mathrm{~nm}$-thick aluminum. A thin layer of hydrophobic photo resist (S1813) was coated on the top of the aluminum layer. The pattern which had been predesigned for the electrical characterization was created by photolithography and development. Then the aluminum at the exposed areas was etched away subsequently and the patterned electrodes were created. The amineterminated SAMs on exposed area between the electrodes were prepared by immersing the substrates into the solution for the formation of (3-Aminopropyl)-triethoxysilane (APTES) SAMs. The solution is composed as follows: $1 \mathrm{~cm}^{3}$ of acetic acid, $1 \mathrm{~cm}^{3}$ of de-ionized water, $25 \mathrm{~cm}^{3}$ of absolute methanol, and $0.5 \mathrm{~cm}^{3}$ of APTES (99\% purity) [6].Then the substrate was immersed in acetone to remove the remained photoresist on the metal electrodes. The processed patterned substrate was rinsed with chloroform and alcohol. Afterward, the substrate with patterned electrodes was immersed into the dispersion of MWCNTs. Modestly hydrophilic amineterminated $\left(-\mathrm{NH}_{2}\right)$ SAMs (contact angle of 30-40 degrees) produce strong attractions to MWCNTs. As a result, the MWCNT was induced to deposit on the surface of amineterminated SAMs. The MWCNTs deposited in the vicinity of the electrodes can bridge two separated electrodes and create MWCNT-based circuit. The MWCNT is hard to be removed just by rinsing because the bonding is strong enough due to the interaction between amine-terminated SAMs and dispersed MWCNT $[7,8]$.

In order to improve the electrical contact between the MWCNT and aluminum electrodes, metal pads were fabricated on the MWCNT using electron beam lithography. A scheme for the fabrication of metal pads on MWCNT is illustrated in Figure 2(b). The detailed process for the fabrication of metal pads on MWCNT can be demonstrated as follows: firstly, it was conducted by spinning single layer of PMMA $950 \mathrm{C} 4$ onto the substrate. The thickness of the PMMA $950 \mathrm{C} 4$ was about $120 \mathrm{~nm}$ controlled according to the curve of thickness versus spin speed. After the ebeam writing, the pattern development was accomplished by soaking the substrate into a mixed solution of MIBK (methyl isobutyl ketone) and isopropanol $(1: 3)$ for 90 seconds and rinsing with isopropanol for 30 seconds. After rinsing and drying, $8 \mathrm{~nm}$ Ti and $50 \mathrm{~nm}$ Au film was evaporated on the substrate by electron beam evaporation method. The substrate was soaked in acetone for 15 minutes to lift-off the Ti/Au and the metal pads on the MWCNT formed.

Figures 3(a) and 3(b) show the FE-SEM pictures of MWCNT-aligned on aluminum electrode patterns at lower and higher magnification, respectively. The MWCNT in the circuit is about $3 \mu \mathrm{m}$ long and placed at the center of the patterned electrodes. The distance of two electrodes is about $2 \mu \mathrm{m}$. The MWNTs are large enough to fill the electrode spacing, allowing for possible current flow. At some other places, several MWCNTs aligned at only one electrode edge and linked up to other electrode. But there were few MWCNT in the area without SAMs. It can be concluded that the SAMs induced the deposition of MWCNT and improve the efficiency of the fabrication of MWCNT-based circuits. 


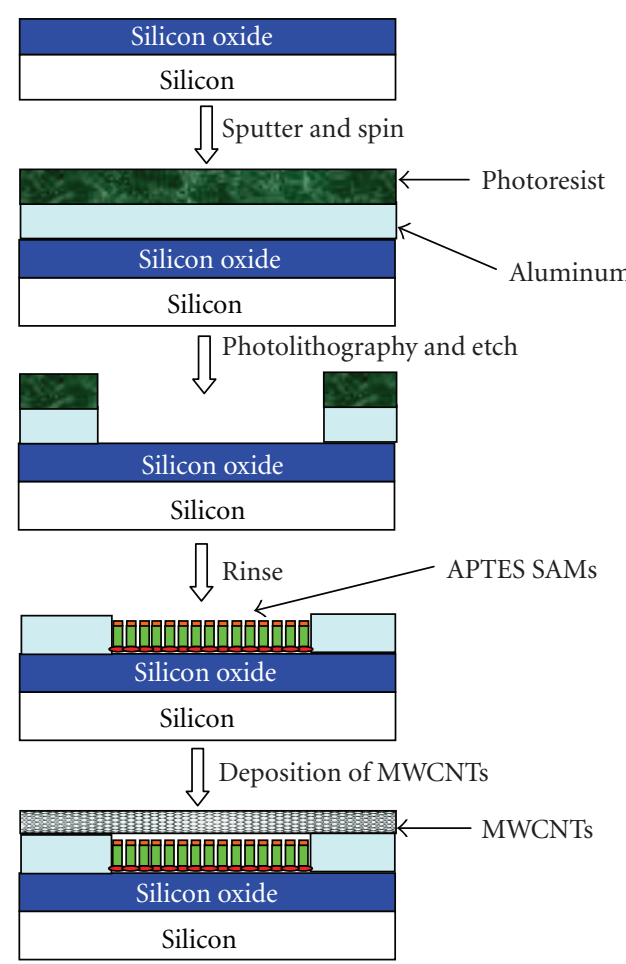

(a)

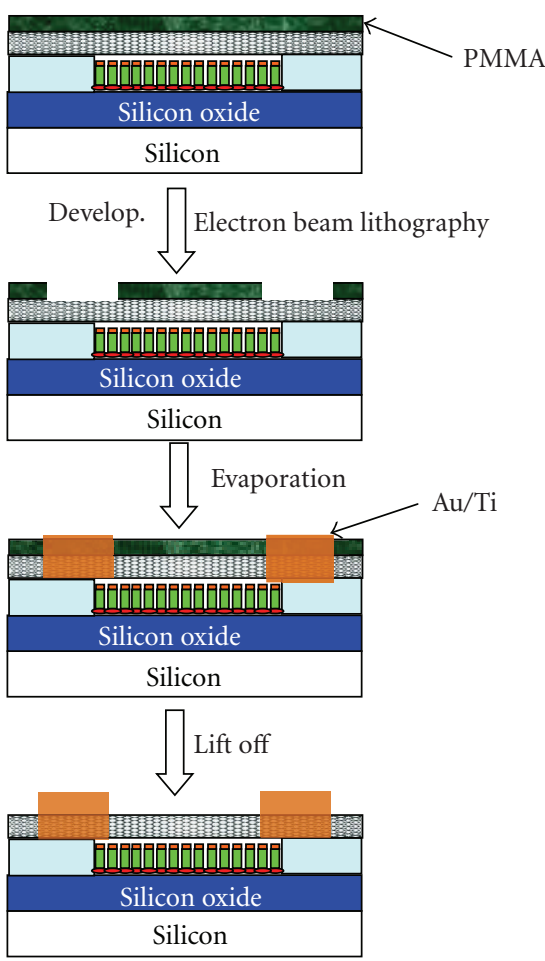

(b)

Figure 2: (a) Scheme of the Deposition of MWCNTs on patterned Aluminum electrode. (b) Fabrication of metal pads on MWCNT.

Figures 3(c) and 3(d) show the SEM image of MWCNTbased circuits after the fabrication of metal pads on MWCNT. The two ends of MWCNT were buried in the Ti/Au metal pads. The contact between MWCNT and electrodes changed from side contact to end contact. The Ti/Au metal pads are more resistant to oxidation and easy to form lower-resistance ohmic contact between MWCNTs and electrodes [9].

Current versus bias voltage $(I-V)$ measurements at room temperature were conducted to evaluate the electrical properties of the placed MWCNT on the prepatterned aluminum electrodes. Figure 4(a) shows the measured current between electrodes in Figures 2(a) and 2(b) as a function of applied voltage. The MWCNT was considered as the main current passing between two electrodes and the resistance of the MWCNT is calculated. The result corresponds to a very high resistance in the order of $P \Omega$. This value is considerably larger than the results previously reported [9]. The MWCNTs do contribute to the current between two electrodes [10,11]. But Aluminum has a low work function at $3.9-4.2 \mathrm{eV}$ and the contacts have equal barriers for both electrons and holes. It is hard to obtain ohmic contact behavior between the CNT and Aluminum interfaces. Another phenomenon associated with this kind of MWCNT-based circuits known as the side contacts, meaning that MWCNTs are electrically contacted only along the circumference of the nanotube. Such contacts result in weak interaction between the carbon and the metal atoms [12]. Generally defect is more easy to form on the outer layer during the purify process of MWCNT. This also may result from the interface between the MWCNT and aluminum which is due to the oxidation of the aluminum before the deposition of MWCNT [13].

Figure 4(b) shows the measured $I-V$ electrical conductance of Figures 3(c) and 3(d) after the fabrication of the metal pads on MWCNT. The current transport between the electrodes shows a nonlinear dependence of applied voltage with the presence of an alumina layer on the aluminum electrodes. It is evident that the electrical pads lying on MWCNTs allow for considerable improvement in the electrical properties of MWCNT-based circuits by many orders of magnitude.

The resistances between the two terminals have been lowered to several $\mathrm{M} \Omega$ by applying contact metal on the top of MWCNTs. The reason for this is very complicated because not only the contact resistance could be lowered but also the multilayers of the MWCNT could contact with the Ti/Au electrodes. The remarkable decrease of the two-terminal resistance has three possible origins as follows: Firstly, Several layers of MWCNT could be carrying the current simultaneously after the MWCNT was buried in the metal pads. The inner layers of MWCNT have better conductive property than the outer layer because the inner layer more perfect crystalline structure. Secondly, the electrical contacts between the MWCNT and the Ti/Au electrodes may improve considerably than $\mathrm{Al}$ electrodes. Finally, the MWCNT may be modified by exposure to PMMA with a resulting resistance decreases [14, 15].

Figure 5 shows the electrical conductance measured between electrodes from $-5 \mathrm{~V}$ through $5 \mathrm{~V}$. The maximum 


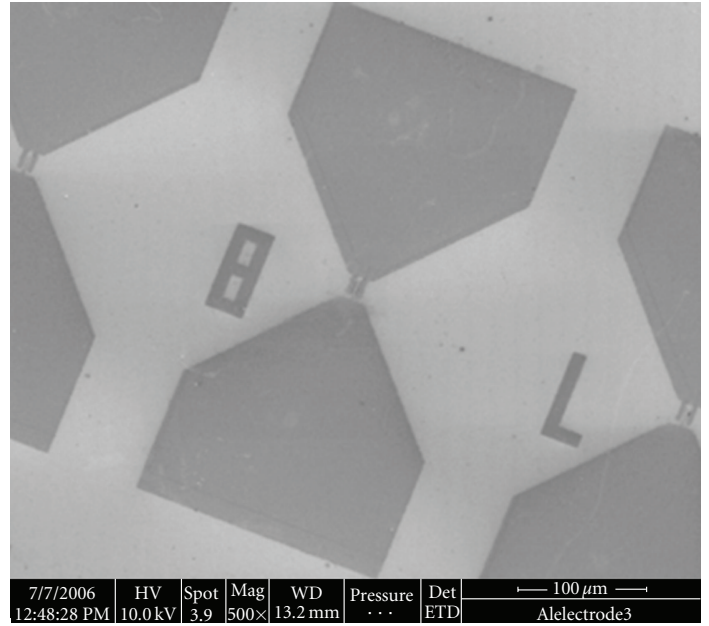

(a)

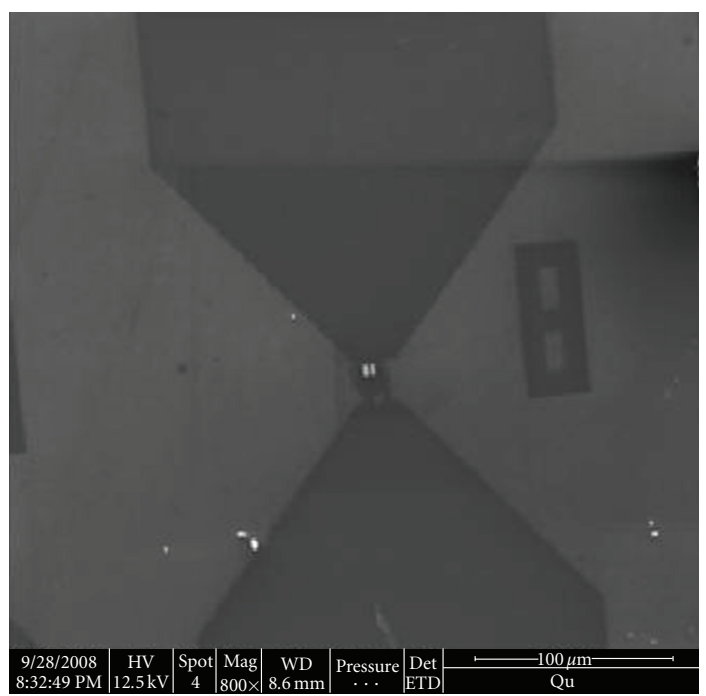

(c)

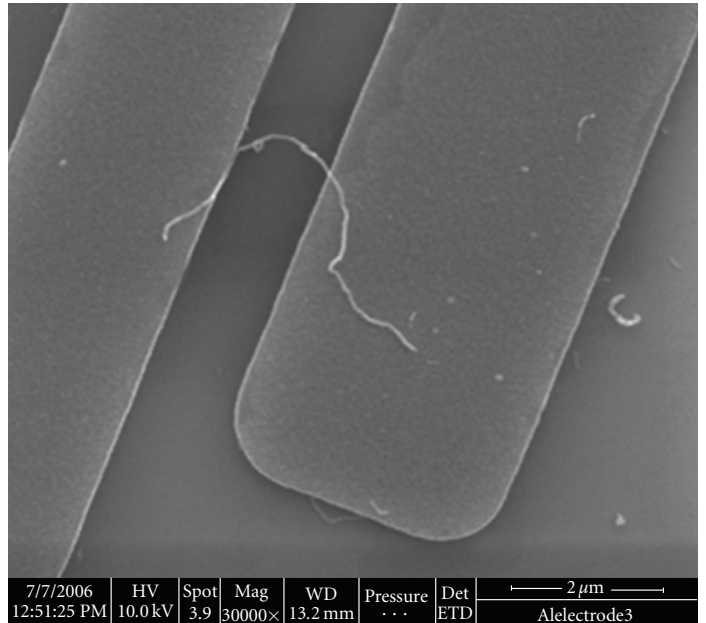

(b)

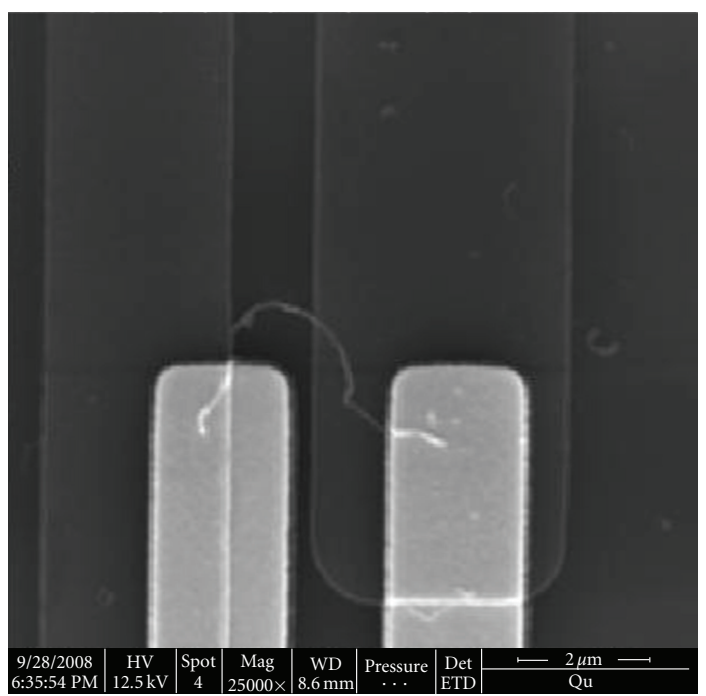

(d)

Figure 3: SEM image of (a) Pattern aluminum electrodes, (b) MWCNT bridging the patterned aluminum electrodes, (c) pattern electrodes with metal pads on MWCNT at low magnification, and (d) MWCNT-based circuits with metal pads on MWCNT at high magnification.

current that the MWCNT stand during the measurement was about $10^{7} \mathrm{~A} / \mathrm{cm}^{2}$ and it is close to the maximum current density of MWCNT reported. It indicates that the electrical contact between the MWCNT and electrodes have been improved by burying the MWCNT into Ti/Au electrodes. Also the resistance can be lowered under larger voltage. The advantage of the technology is to construct devices out of preselected MWCNT which will provide a possibility to build a great variety of complex CNT-based devices.

\section{Conclusion}

The MWCNTs were induced and placed on prepatterned aluminum electrodes by self-assembled monolayers to fabricate the MWCNT-based circuit. This process improved the efficiency of fabrication of MWCNT electronic circuits. Then metal pads on MWCNT were fabricated by electron beam lithography method. The electrical properties of MWCNT-based circuits were characterized with two probe system before and after the fabrication of metal pads on MWCNT. Electrical properties of MWCNT-based circuits were improved largely after two metal pads were fabricated on the MWCNT.

\section{Acknowledgments}

This work is supported by State Key Laboratory of Mechanical Transmission, Chongqing University (Grant no. SKLMTKFKT-200904). This work also is supported partly by the Natural Science Foundation of Jiangsu Province under grant No. bk2009265, in part by the New Century Training Program Foundation for the Talents by the State Education Commission under Grant no. NCET080107, in part by the Key Project of Chinese Ministry of Education under 


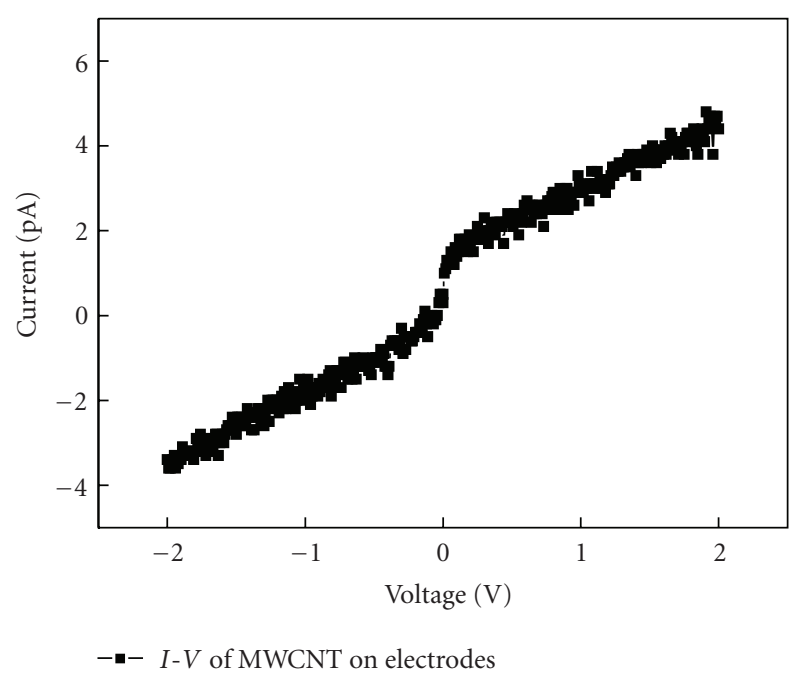

(a)

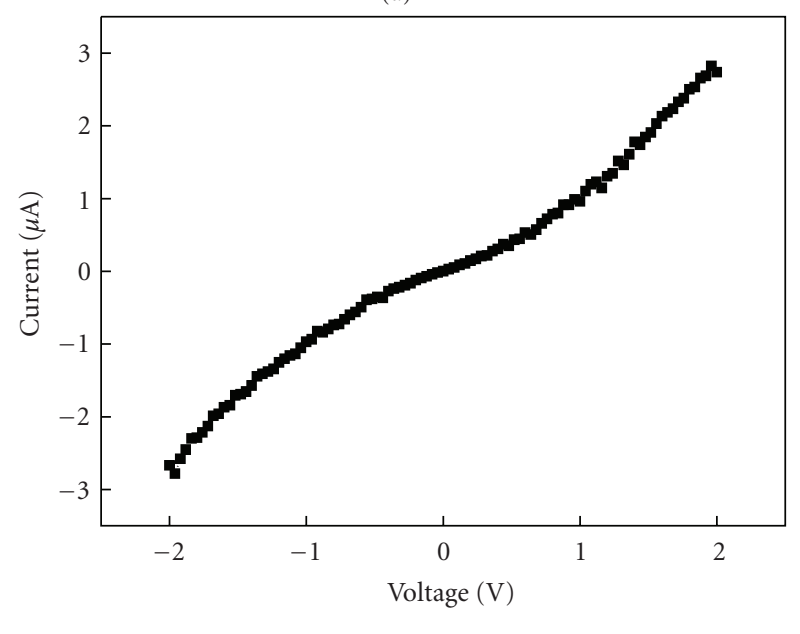

$--I-V$ of MWCNT-based circuit

(b)

FIGURE 4: $I-V$ behavior of MWCNTs-based circuits before (a) and after (b) the fabrication of metal pads on MWCNT.

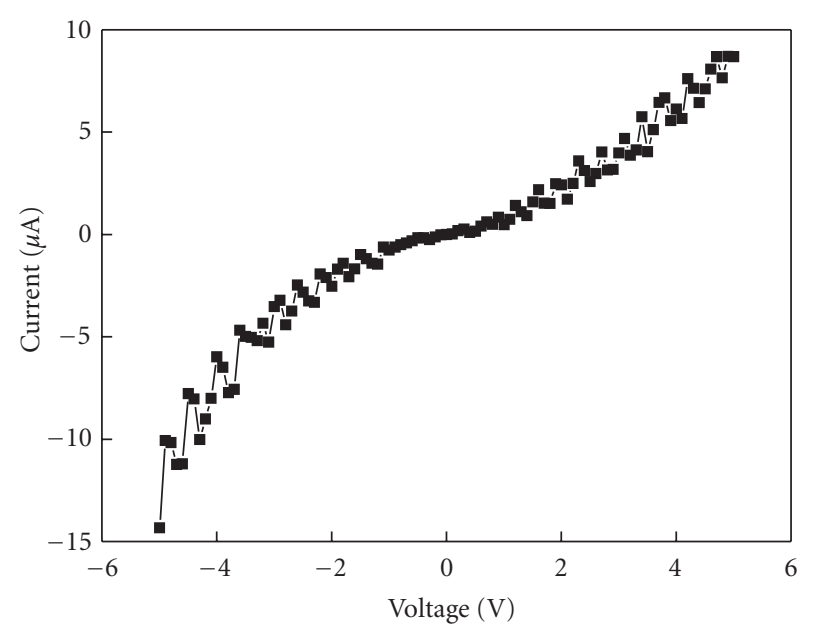

$-\square-I-V$ of MWCNT-based circuit

FIGURE 5: $I-V$ behavior of MWCNTs-based circuits that the voltage varied from $-5 \mathrm{~V}$ to $5 \mathrm{~V}$.
Grant no. 109074, in part by National Aerospace Science Foundation of China 20080169002.

\section{References}

[1] L. A. Nagahara, I. Amlani, J. Lewenstein, and R. K. Tsui, "Directed placement of suspended carbon nanotubes for nanometer-scale assembly," Applied Physics Letters, vol. 80, no. 20, pp. 3826-3829, 2002.

[2] S. Lu, J. Chung, and R. S. Ruoff, "Controlled deposition of nanotubes on opposing electrodes," Nanotechnology, vol. 16, no. 9, pp. 1765-1770, 2005.

[3] J. Liu, M. J. Casavant, M. Cox et al., "Controlled deposition of individual single-walled carbon nanotubes on chemically functionalized templates," Chemical Physics Letters, vol. 303, no. 1-2, pp. 125-129, 1999.

[4] M. Burghard, G. Duesberg, G. Philipp, J. Muster, and S. Roth, "Controlled adsorption of carbon nanotubes on chemically modified electrode arrays," Advanced Materials, vol. 10, no. 8, pp. 584-588, 1998.

[5] X. M. H. Huang, R. Caldwell, L. Huang et al., "Controlled placement of individual carbon nanotubes," Nano Letters, vol. 5, no. 7, pp. 1515-1518, 2005.

[6] Y. Peng, Y. Hu, and H. Wang, "Patterned deposition of multiwalled carbon nanotubes on self-assembled monolayers," Chinese Science Bulletin, vol. 51, no. 2, pp. 147-150, 2006.

[7] K. H. Choi, J. P. Bourgoin, S. Auvray et al., "Controlled deposition of carbon nanotubes on a patterned substrate," Surface Science, vol. 462, no. 1, pp. 195-202, 2000.

[8] J. C. Lewenstein, T. P. Burgin, A. Ribayrol, L. A. Nagahara, and R. K. Tsui, "High-yield selective placement of carbon nanotubes on pre-patterned electrodes," Nano Letters, vol. 2, no. 5, pp. 443-446, 2002.

[9] P. S. Na, H. Kim, H.-M. So et al., "Investigation of the humidity effect on the electrical properties of single-walled carbon nanotube transistors," Applied Physics Letters, vol. 87, Article ID 093101, 3 pages, 2005.

[10] M. H. Yang, K. B. K. Teo, W. I. Milne, and D. G. Hasko, "Carbon nanotube Schottky diode and directionally dependent field-effect transistor using asymmetrical contacts," Applied Physics Letters, vol. 87, no. 25, Article ID 253116, 3 pages, 2005.

[11] H. Watanabe, K. Shimotani, T. Shigematu, and C. Manabe, "Electric measurements of nano-scaled devices," Thin Solid Films, vol. 438-439, pp. 462-466, 2003.

[12] K. Ojima, Y. Otsuka, T. Matsumoto, T. Kawai, K. Nakamatsu, and S. Matsui, "Printing electrode for top-contact molecular junction," Applied Physics Letters, vol. 87, no. 23, Article ID 234110, pp. 1-3, 2005.

[13] A. Bachtold, M. Henny, C. Terrier et al., "Contacting carbon nanotubes selectively with low-ohmic contacts for four-probe electric measurements," Applied Physics Letters, vol. 73, no. 2, pp. 274-276, 1998.

[14] J. Chung and J. Lee, "Nanoscale gap fabrication and integration of carbon nanotubes by micromachining," Sensors and Actuators A, vol. 104, no. 3, pp. 229-235, 2003.

[15] T. Shimizu, H. Abe, A. Ando, Y. Nakayama, and H. Tokumoto, "Electrical conductivity measurements of a multi-walled carbon nanotube," Surface and Interface Analysis, vol. 37, no. 2, pp. 204-207, 2005. 

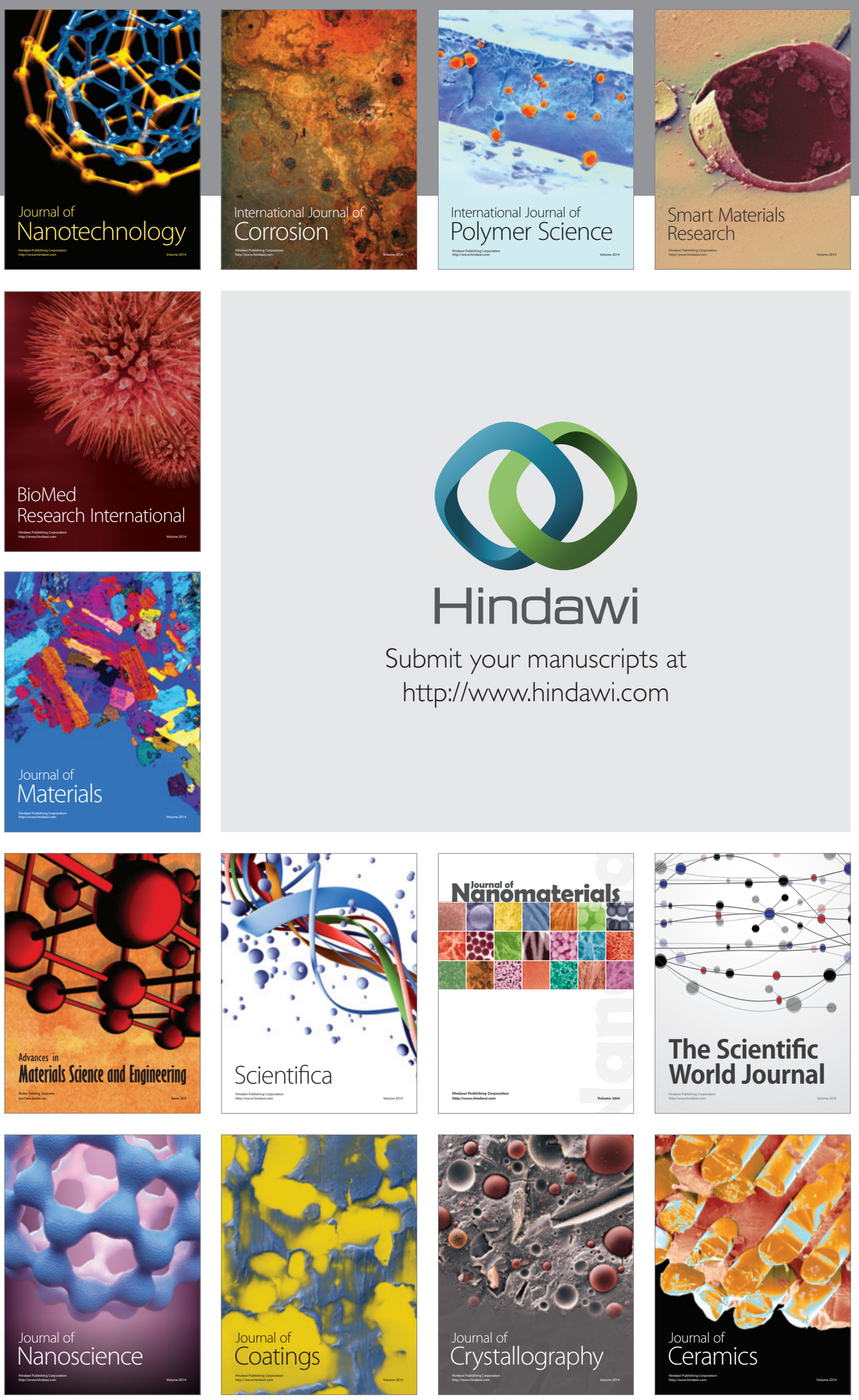

The Scientific World Journal

Submit your manuscripts at

http://www.hindawi.com

\section{World Journal}

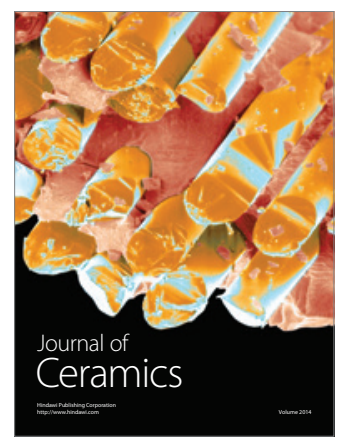

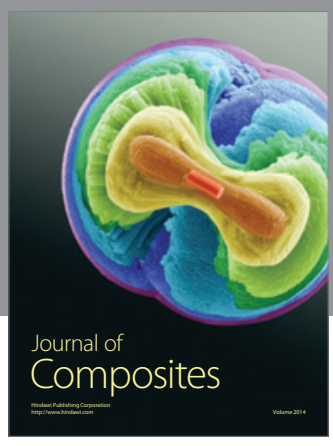
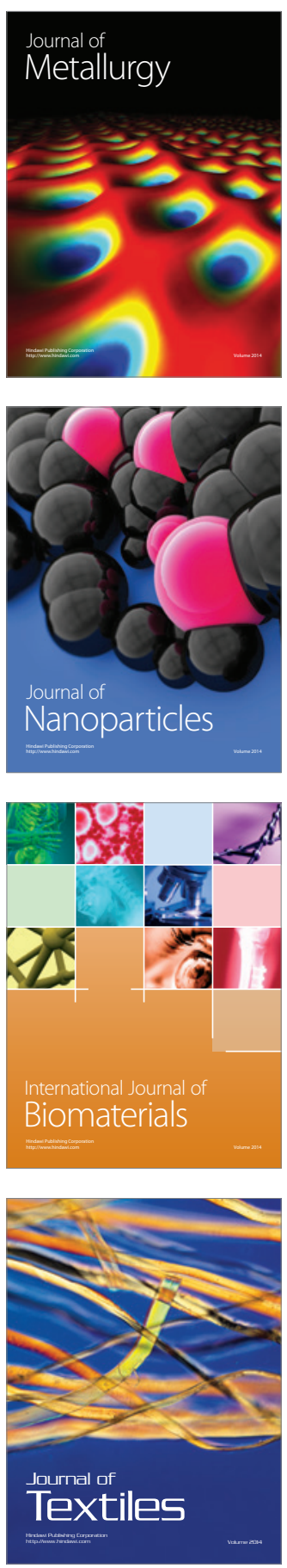